\title{
TWO-PHASE FLOW IN HELE-SHAW CELLS: NUMERICAL STUDIES OF SWEEP EFFICIENCY IN A FIVE-SPOT PATTERN
}

\author{
LEONARD W. SCHWARTZ ${ }^{1}$ AND ANTHONY J. DEGREGORIA ${ }^{2}$
}

(Received 19 December 1986; revised 6 April 1987)

\begin{abstract}
The unsteady Hele-Shaw problem is a model nonlinear system that, for a certain parameter range, exhibits the phenomenon known as viscous fingering. While not directly applicable to multiphase porous-media flow, it does prove to be an adequate mathematical model for unstable displacement in laboratory parallel-plate devices. We seek here to determine, by use of an accurate boundary-integral front-tracking scheme, the extent to which the simplified system captures the canonical nonlinear behavior of displacement flows and, in particular, to ascertain the role of noise in such systems. We choose to study a particular pattern of injection and production "wells." The pattern chosen is the isolated "five-spot," that is a single source surrounded by four symmetrically-placed sinks in an infinite two-dimensional "reservoir." In cases where the "pusher" fluid has negligible viscosity, sweep efficiency is calculated for a range of values of the single dimensionless parameter $\tau$, an inverse capillary number. As this parameter is reduced, corresponding to increased flow rate or reduced interfacial tension, this efficiency decreases continuously. For small values of $\tau$, these stable displacements change abruptly to a regime characterized by unstable competing fingers and a significant reduction in sweep efficiency. A simple stability argument appears to correctly predict the noise level required to transit from the stable to the competing-finger regimes. Published compilations of experimental results for sweep efficiency as a function of viscosity ratio showed an unexplained divergence when the pusher fluid is less viscous. Our simulations produce a similar divergence when, for a given viscosity ratio, the parameter $\tau$ is varied.
\end{abstract}

\footnotetext{
${ }^{1}$ Department of Mechanical and Aerospace Engineering, Rutgers University, New Brunswick, New Jersey 08855-0909, U.S.A.

Present address: Department of Mechanical Engineering, University of Delaware, Delaware, DE 19716, U.S.A.

${ }^{2}$ Corporate Research Science Laboratories, Exxon Research and Engineering Co., Clinton Township, Route 22E, Annandale, New Jersey 08801, U.S.A.

Present address: Astronautics Corp. of America, Madison, Wisconsin, U.S.A.

(C) Copyright Australian Mathematical Society 1988, Serial-fee code 0334-2700/88
} 


\section{Introduction}

A Hele-Shaw cell is a simple laboratory device consisting of two plates of glass separated by a small constant distance. A displacement process where, for example, a more viscous fluid is pushed out by a less viscous one may thus be visualized. Some time agn it was shown [1], [19] that this process is inherentiy unstable and, provided the cell is of sufficient size, an initially straight interface between the fluids will develop undulations. When these undulations grow to large amplitude, the less viscous fluid may be observed to form "fingers" into the more viscous one; this is the celebrated viscous fingering instability. A variant of the Hele-Shaw cell is the sand-pack or bead-pack where the space between the plates is filled with a packing of small particles. Viscous fingering has also been observed in these devices when the "pusher" fluid is less viscous [4]. Unstable displacements can be divided into two classes: they are called immiscible when the fluid interface is sharp and possesses a finite value of interfacial tension; else they are termed miscible.

Hele-Shaw cells have found application in the oil industry [4] because they are (grossly) simplified models that illustrate the flow behavior when viscous oil is pushed out by a less viscous fluid such as water. In this context, a canonical problem may be formulated. We specify one or several points where water is injected into the cell and some points where oil is "produced." Assuming that the two phases are immiscible and that water completely replaces oil as the front passes a given point, that is the "displacement efficiency" is unity, we wish to determine or optimize the total amount of oil produced before the water "breaks through" to a production well. Once a continuous water path is formed from an injector to a producer, subsequent production will consist largely of water, since the total resistance to flow along this path is very low for large values of the ratio of oil to water viscosities. For unit displacement efficiency, the quantity of oil produced is simply the portion of the cell contacted by the water prior to break-through. Assuming the cell or "reservoir" to be two-dimensional, this fraction is termed the areal sweep efficiency.

Mathematically, the problem to be solved is nonlinear with a moving interface whose time history needs to be determined as part of the solution. Under a set of a priori reasonable assumptions, [6], [22] we have a velocity field given as the solution of the two-dimensional Laplace equation within each phase, that is

$$
\begin{gathered}
\mathbf{v}=-(k / \mu) \nabla p \\
\nabla \cdot \mathbf{v}=-(k / \mu) \nabla^{2} p=0,
\end{gathered}
$$

where $p$ is pressure, $\mu$ the constant fluid viscosity and $k$ is the constant permeability which, for a Hele-Shaw cell, is equal to $b^{2} / 12$ where $b$ is the spacing 
between the plates. The pressure jump across the interface is taken as $\sigma \kappa$ where $\sigma$ is the interfacial tension and $\kappa$ is the curvature of the interface when the cell is viewed from above. Viscous contributions to the pressure jump are neglected, as is the variation in the curvature component between the plates. We shall treat a number of cases where the driver fluid has negligible viscosity; under this assumption, the pressure within the driver may be taken equal to zero without loss of generality.

A number of laboratory studies of viscous fingering have been performed in the past thirty years. While many of these employ a linear channel, several investigators have used geometries related to that studied here. In miscible displacements at large Peclet number, destabilising convective effects dominate and rather sharp phase fronts are observed experimentally. Notable among these miscible experiments are the works of Habermann [7], who performed sand-pack floods, and Lee \& Claridge [11], who used a Hele-Shaw apparatus. In both cases, the well geometry was one-quarter of a developed five-spot pattern. This geometry has many features in common with the isolated five-spot studied here. An immiscible Hele-Shaw experiment was recently reported by Paterson [17], who used a radial flow geometry with injection of the displacing fluid at the center of the apparatus. In all these experiments, fingering is observed that is caused by some type of noise in the experimental set-up. In the Paterson experiment, the fingering arises via a linear instability mechanism and is stimulated, in principle, by infinitesimal disturbances. In the five-spot geometry, on the other hand, a critical disturbance level, depending on flooding speed, needs to be exceeded before fingering instabilities become important. This nonlinear effect will be demonstrated numerically below. In this regard, the five-spot or, for that matter, any pattern with discrete production wells in the finite plane, is similar to the linear channel case for which we have established [5], [6] that the relevant instability mechanism is nonlinear. Unlike the case of a long rectilinear channel where the repeated finger-tip splitting does not appear to greatly affect the average value of finger width, here the nonlinear instability may produce a large reduction in the areal sweep efficiency.

Section 2 is devoted to problem formulation. The induced velocity, at any instant of time, is found from the shape of the flood front as the solution of a singular integral equation. In Section 3 we treat the numerical implementation of the front-tracking method as specialized to the five-spot pattern. When the pusher fluid viscosity is equal to zero, breakthrough sweep efficiencies are calculated for a range of values of capillary number. At relatively low speeds, numerical results yield breakthrough profiles with only a single "finger" in each five-spot quadrant. These "stable" solutions correspond to relatively large values of sweep efficiency. At high speeds, or at lower speeds where random noise is introduced into the calculation, as discussed in Section 4, finger break-up is 
observed. By comparing the magnitude of the asymmetry caused by the remote production wells with the initial rate of growth of unstable disturbance modes, a critical noise amplitude may be estimated analytically. For a particular value of capillary number, we have introduced white noise into our numerical solution and have monitored its effect on fingering patterns and areal sweep efficiency. Even though our analytical estimate uses only information known at the start of a flood, it seems to correctly predict the noise level required for measurabie changes in sweep efficiency. By a simple argument we associate noise level with permeability variation. We find that the critical value of heterogeneity for transition to floods that are dominated by the unstable modes is surprisingly low.

In Section 5, results are extended to finite-viscosity-ratio cases. Sweep efficiencies are higher, as expected. Both stable and unstable displacements are considered, as well as the dependence of sweep efficiency on flooding speed. For cases where the displacement front is not destabilised by noise, greater efficiency arises simply from the increased flow resistance in the driving phase. When the driver is less viscous and noise is introduced, the sensitivity of the front to noise is reduced, and significantly larger disturbance levels can be tolerated before the front breaks up into a multiply-fingered pattern.

In the final section we summarize our findings and relate our conclusions to the results of others. We also briefly discuss the connection with the general question of pattern formation in nonlinear systems.

\section{Problem formulation}

Recently we have developed a boundary integral technique to treat these HeleShaw problems. The basic method is described in more detail elsewhere [5], [6]; here we will focus primarily on the implementation of the method to a particular pattern of injection and production wells. Specifically we treat the isolated inverted five-spot pattern, a single injection well surrounded by four symmetricallyplaced production wells in a two-dimensionally infinite cell or reservoir.

The Hele-Shaw equations with finite viscosity ratio can be written as

$$
\nabla^{2} p_{i}=0, \quad(x, y) \in D_{i}, i=1,2,
$$

where $D_{1}$ is the domain occupied by the displaced fluid and $D_{2}$ corresponds to the displacing phase. The velocity potentials are related to the pressures $p_{i}$ by

$$
\phi_{i}=-\left(k / \mu_{i}\right) p_{i} .
$$

The permeability $k$ for a Hele-Shaw cell is $b^{2} / 12$, where $b$ is the plate spacing. At the interface $\partial D$, the continuity equations are

$$
p_{1}-p_{2}=\sigma \kappa
$$


and

$$
\partial \phi_{1} / \partial n=\partial \phi_{2} / \partial n
$$

Here $\kappa$ is the curvature of the interface in the plane of the cell and $\sigma$ is the interfacial tension. The kinematic condition determining the interface motion is

$$
\left(x_{t}, y_{t}\right) \cdot \mathbf{n}=\partial \phi / \partial n
$$

For the five-spot pattern, the problem is nondimensionalized by using the interwell distance $L$ as reference length and $Q$, the areal flux in one-quarter of the pattern, as reference potential. The viscosity ratio

$$
m=\mu_{2} / \mu_{1}
$$

is less than one for unstable floods. The mobility ratio $M$, as usually defined, is the reciprocal of $m$. The geometry is shown in Figure 1.

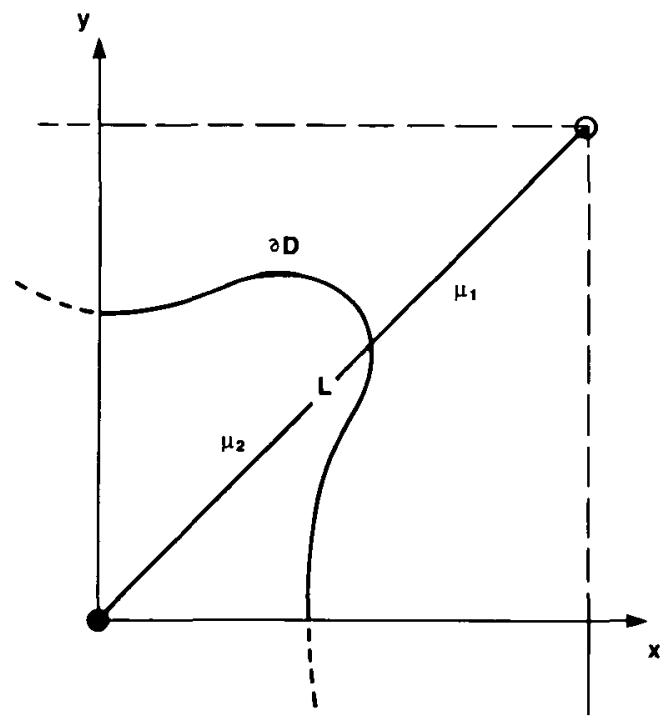

Figure 1. One-quarter of an isolated five-spot pattern. The general direction of flow is from lower left to upper right.

The remainder of the derivation, at this point, will pertain to the case $m=0$. Specific extensions for finite viscosity ratios will be given in Section 5 . Thus here $p=p_{1}, \mu=\mu_{1}$ and $p_{2}=\mu_{2}=0$ without loss of generality. Measured in units of $12 Q \mu / b^{2}$, the dimensionless pressure satisfies

$$
p=\tau \kappa, \quad z \in \partial D,
$$

subject to the kinematic boundary condition, in complex notation,

$$
\partial z / \partial t=\overline{d f} / d z, \quad z \in \partial D .
$$


Except for initial conditions, the time history of the motion is seen to depend only on the single dimensionless parameter

$$
\tau=\sigma b^{2} / 12 \mu Q L,
$$

where $Q$ is the constant areal flux or production rate at each of the sinks. Overall continuity requires that the injection rate at the source be equal to 4 in dimensionless units. In equation (7), $f$ is the complex potential $\mu+i \psi$ and the bar signifies complex conjugation. Note that only the surface-normal component of (7) need be satisfied. Four-fold reflectional symmetry of the flow is assumed. This is motivated both by a desire to contain the size of the computational problem and also to allow comparison with experiment where investigators typically model only one quadrant of the five-spot pattern. It should be emphasized that we do not address here actual problems of oil production. Rather our goal is to investigate the mathematical behaviour of this simplified model and determine to what extent complex fingering patterns, such as those seen in experiments, can be produced.

With the assumed symmetry, we find it convenient to map the fluid domain into a compact region of the $\zeta$-plane using $\zeta=1 / z^{2}$ where the flow pattern is symmetric about the $\xi(=\operatorname{Re} \varsigma)$ axis. Having determined the boundary pressures in the $z$-plane using equation (6), we solve for the boundary source distribution $q(s)$ in the 5 -plane by

$$
\int_{\partial D_{\varsigma}} q\left(s_{1}\right) \ln \left|\varsigma-\varsigma_{1}\right| d s_{1}=2 \pi p(s)-\ln \left|\varsigma^{2}+1\right|, \quad \varsigma \in D_{\varsigma}
$$

where vertical bars denote the modulus of a complex number. Having determined $q$, the induced velocity at a point $\zeta$, approaching $\partial D_{\varsigma}$ from within, is given by

$$
\frac{d f}{d \varsigma}=u^{(\varsigma)}-i v^{(\varsigma)}=-\frac{\varsigma}{\pi\left(\varsigma^{2}+1\right)}+\frac{1}{2 \pi} \int \frac{q\left(s_{1}\right)}{\zeta-\varsigma_{1}} d s_{1}-\frac{i q(s)}{2} e^{-i \beta} .
$$

Here $\beta$ is the inclination of a segment of $\partial D_{\varsigma}$ (the image of $\partial D$ ) measured counterclockwise from the positive $\xi$ axis and the integral is of Cauchy-principalvalue type. Finally the boundary velocities are transformed back to the physical plane using

$$
\frac{d f}{d z}=\frac{d f}{d \varsigma} \frac{d \varsigma}{d z}
$$

\section{Numerical implementation and results for inviscid driver}

The numerical treatment of equations (9) and (10) is described in some detail in reference [6]. Briefly, the surface is represented by $2 N+1$ points or nodes 
in the $s$-plane and $q$ is taken to be piecewise constant. Because of the assumed symmetry in the $z$-plane, however, the discrete version of equation (9a) is only of dimension $N$. Having, in effect, reduced the problem to a system of first-order nonlinear ordinary differential equations, the time evolution of the interface is calculated implicitly using the LSODE [8] package with sufficiently small values of error tolerance.

The power of the boundary-integral method for problems of this type can be fully exploited by redistribution of the boundary nodes so as to cluster points in regions of large potential gradients. At each time step the points are redistributed along a cubic-spline representation of the boundary using the algorithm

$$
\Delta s=\operatorname{Min}\left(A \kappa, B(\tau / v)^{1 / 2}, C\right)
$$

where $\Delta s$ is the distance between adjacent points in the $z$-plane and $v$ is the magnitude of the velocity. Parameters $A$ and $C$ are selected so as to provide reasonable definition of the interface. More critical is the choice of $B$ which is dictated by local stability considerations. Too large a value of $B$ will, in effect, introduce an artificial surface tension that prevents finger splitting. Too small a value not only increases the dimension of the linear system to be solved but may, in addition, cause the unsteady problem to become 'stiff,' that is, having widely disparate eigenvalues; when the problem is stiff, computer run times increase dramatically. For most of the cases discussed here, we used $0.5,0.75$, and 0.05 for $A, B$, and $C$ respectively.

Two different five-spot patterns are discussed in the literature. Our isolated five-spot may be contrasted with the developed five-spot pattern which is an infinite double-periodic array of sources and sinks where each singularity is surrounded by four symmetrically placed singularities of the other kind. For the developed pattern, the sides of each square thus formed must be streamlines. This is not the case for the isolated pattern. Both patterns are idealizations of actual well configurations and the isolated case has the advantage that the conformal map from the $z$ to the $\varsigma$ planes is quite simple; the corresponding map for the developed pattern would require many numerical evaluations of elliptic functions with complex argument, which could add significantly to computer usage.

For reference purposes, we shall calculate the frontal history of the isolated five-spot assuming single-phase flow. The complex potential is simply

$$
f=(1 / 2 \pi) \log \left[z^{4} /\left(z^{4}+1\right)\right]
$$


Solving for $z(f)$, the travel time from the central source along a particular streamline $\psi$ to a point where the potential is $p$ may be written as

$$
\begin{aligned}
t & =\int_{-\infty}^{p}\left|\frac{d z}{d f}\right| d \phi \\
& =\frac{\pi^{2}}{4} \int_{-\infty}^{p} \frac{e^{\pi \phi} d \phi}{\left(1+e^{4 \pi \phi}-2 e^{2 \pi \phi} \cos 2 \pi \psi\right)^{5 / 4}} .
\end{aligned}
$$

The shape of the front at a given time $t$ is then calculated by numerical quadrature of (13) and use of quadratic inverse interpolation to find corresponding points in the $z$-plane. The single-phase fronts are shown in Figure 2.

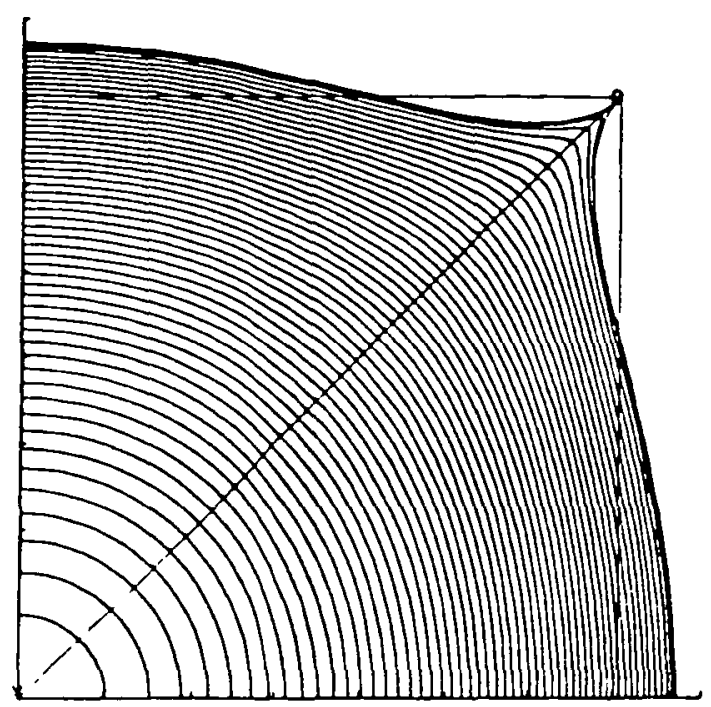

Figure 2. Frontal history of a five-spot in single-phase flow, time interval $\Delta t=0.01$.

This figure may be compared with the single-phase flood history for the developed five-spot determined experimentally by Muskat [15] using electrolytic models. For our isolated pattern, the breakthrough time is easily calculated to be $\pi / 6$. Since the breakthrough time is proportional to the flooded area, this corresponds to a contacted area equal to about 105 per cent of the area enclosed by the square in Figure 1. We shall use this well pattern area as the reference area in calculating sweep efficiency.

The single-phase flood result may be compared with the boundary-integral simulation of flow at infinite (unfavorable) viscosity ratio as shown in Figure 3. The value of $\tau$ for this case is 0.001 . This calculation was started with equallyspaced points on a quarter circle of radius $r_{0}=0.1$. Assuming circular symmetric source flow until this radius is reached, the travel time from the source is 
$7.85 \times 10^{-3}$. Successive time profiles are shown for time increments $\Delta t$ equal to 0.010 until the closest point on the front is within a distance of 0.2 of the sink. Successively smaller time intervals are then used so that the breakthrough time can be estimated to at least three significant figures. Here the time of breakthrough is 0.368 corresponding to an areal sweep efficiency of 73.6 per cent. The flood chooses an essentially symmetric pattern even though symmetry about the diagonal is not imposed. We have repeated the calculation of Figure 3 with symmetry imposed. This is conveniently accomplished by using the conformal map

$$
\varsigma=1+1 / z^{4}
$$

The corresponding flow in the $\varsigma$-plane is bilaterally symmetric with a single sink at the origin. The flow pattern is virtually identical to Figure 3 and the sweep efficiency agrees with the previous result to within 0.2 per cent. For the case without imposed symmetry, the number of points used to describe the front increased from 17 at $r_{0}=0.1$ to a maximum of 43 at the $t=0.31$ profile. Point redistribution was performed along each output profile using formula (11). Because redistribution changes the discrete problem, the choice of time intervals influences the subsequent flood history. In all cases, time intervals were chosen sufficiently small so that errors, from this source, may be neglected. The calculation shown in Figure 3 required 40 sec on an IBM 3033 computer.

To establish convergence of the solution as the resolution of the interface is increased, a series of runs were made at $\tau=10^{-3}$ with various values of the pointspacing parameter $B$. In Table I we show computed values of breakthrough sweep efficiency versus $B$. Examination of this sequence suggests that the convergence

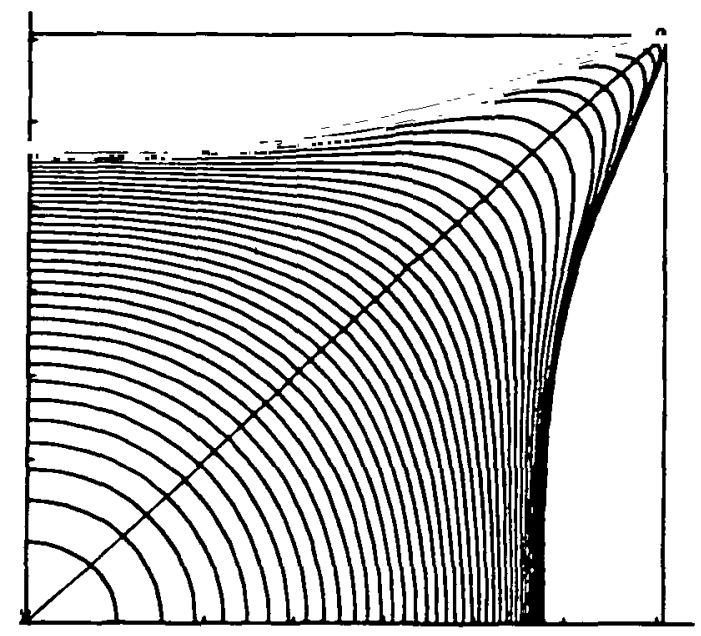

Figure 3. A calculated stable sweep for $\tau=0.001, \Delta t=0.01$. 
of the sweep efficiency is approximately quadratic in $B$ and that $B=0.75$ is sufficiently small so that an accuracy claim of better than one per cent is justified. Tests at other values of $\tau$ show similar rates of convergence. In the sequel we will consistently use $B=0.75$ which is equivalent in most cases to requiring that the number of points used to describe a profile scales as $\tau^{-1 / 2}$.

TABLE I. Convergence of Numorical Solütion. Vai iáliun of sweep efficiency $\eta$ with pointspace parameter $B$.

\begin{tabular}{l|l}
\multicolumn{1}{c|}{$B$} & \multicolumn{1}{c}{$\eta$} \\
\hline 3.0 & $77.0 \%$ \\
1.5 & 75.0 \\
0.75 & 73.6 \\
0.375 & 73.2
\end{tabular}

\section{The effect of noise on sweep efficiency for an inviscid driver}

We wish now to consider the effect of noise on "stable" solutions such as that shown in Figure 3. We require, for later use, the result of a linear stability analysis for a slightly perturbed circular front that is expanding at constant areal velocity. Let the shape of this interface by given by

$$
R(\theta, t)=R_{0}(t)+\varepsilon e^{\omega t} \sin n \theta
$$

for integer values of $n$ and $\varepsilon \ll 1$. The potential $p$ at time $t$ is given by

$$
-p=(2 Q / \pi) \log \left(r / R_{0}\right)+b^{2} \sigma /\left(12 \mu R_{0}\right)+C\left(R_{0} / r\right)^{n} \sin n \theta
$$

where $C$ is order $\varepsilon$ and higher-order corrections have been neglected. Applying now the pressure-jump and kinematic boundary conditions, with the curvature approximated to $0(\varepsilon)$, we readily arrive at the result

$$
\omega_{n}=\frac{(n-1)}{R_{0}^{2}}\left[\frac{2 Q}{\pi}-\frac{n(n+1) b^{2} \sigma}{12 \mu R_{0}}\right]
$$

for the growth of the $n$th disturbance mode. An essentially similar expression is derived by Wilson [24] using a somewhat different method. Equation (17) reveals that a sufficiently small circle is stable to all small perturbations, and that successively higher modes become unstable as $R_{0}$ increases.

For $R_{0}$ small, the effect of the remote sinks can also be treated as a perturbation to the expanding circular front by approximating the flow field, given by equation (9), for $|z| \ll 1$. We wish to compare the growth rate of the asymmetry caused by the suction of the remote wells with the rate of growth of the most unstable noise-induced eigenmode as given by equation (17). The rationale for considering these competing processes follows from the results of our previous 
study on flow in a linear channel, [5], [6] i.e. the standard Saffman-Taylor geometry [19]. There it was established, by both numerical results and an heuristic analytical model, that effective stability of developed fingers to small perturbations rests on the observation that disturbances initiated near the finger tip are convected backward by the dilation of the interface. While certain disturbance modes will grow initially, they are ultimately "left behind" in the viscous liquid as the finger tip progresses down the channel. Similarly, in the present case, the four-lobed suction pattern will tend to draw the interface away from the noisy perturbations. Only if the order of noise growth exceeds the rate of development of the stable four-lobed pattern, can unstable fingering be expected. Just as in the linear channel, we believe the patterns of the type shown in Figure 3, are stable to low-level noise and (nonlinearly) unstable to noise of sufficient amplitude. Thus this comparison of competing processes may be used to provide an order-of-magnitude estimate of the critical noise level.

Combining these competing effects, we obtain the initial rate of growth of a perturbed circle as

$$
\frac{\partial R}{\partial t}=\frac{2 Q}{\pi R_{0}}-\frac{2 Q R_{0}^{3}}{\pi L^{4}} \cos 4 \theta+\varepsilon \omega_{n} \sin n \theta
$$

The last two terms will be of comparable magnitude if

$$
2 R_{0}^{3} Q /\left(\pi L^{4} \varepsilon \omega_{n}\right)=C
$$

and $C$ is order one. For ease of subsequent manipulation we take $C$ equal to one and obtain, for the critical $n$th mode disturbance amplitude

$$
\hat{\varepsilon}_{n}^{*}=\hat{R}_{0}^{5}\left\{(n-1)\left[1-\pi n(n+1) \tau /\left(2 \hat{R}_{0}\right)\right]\right\}^{-1}
$$

where $\hat{\varepsilon}=\varepsilon_{n} / L, \hat{R}_{0}=r_{0} / L$. These critical amplitudes are seen to decrease with decreasing $\hat{R}_{0}$ and decreasing $\tau$ as expected. When the term in square brackets in either equation (17) or (20) is equal to zero, we have the condition for neutral growth of small perturbations. The point-spacing criterion, corresponding to the second term on the right side of equation (11), is derived from the neutral growth condition. Taking the constant $B$ equal to 0.75 corresponds to using about eight points per neutrally stable wavelength based on local conditions. The effective value is somewhat larger, however, because of the surface dilation that occurs between point redistribution time intervals.

To assess the effect of noise, associated with spatially varying permeability, for example, we purposefully introduce slight displacements to the surface nodes at each output time. A given coordinate $x_{0}$, say, is moved to a new position $x$ according to

$$
x=x_{0}+\delta A v
$$

where $v$ is the local speed, $A$ is a random number lying in the interval $(-1,1)$ and $\delta$ is a global input parameter called the noise level. A relation between the 
noise level and the variation in permeability follows from a simple argument. In a given (small) time interval $\Delta t$, we consider the displacement of a fluid particle $\Delta x$. With a given value of pressure gradient, $\Delta x$ will be proportional to the permeability $k$. An incremental change in $\Delta x$ will be proportional to a permeability increment $\delta k$, say. Thus $\delta k / k \approx A v \delta /(v \Delta t)$; since the average value of the magnitude of $A$ in either the forward or backward directions is $\langle|A|\rangle=0.5$, we may calculate the average fractional permeability variation as

$$
\langle\delta k / k\rangle=\delta /(2 \Delta t)
$$

Some justification is required for our procedure of introducing noise only on the fluid interface rather than within the fluid region as well. Since the effect of permeability variation is to displace a fluid particle by an amount proportional to its velocity, we wish to establish that the velocity change associated with a small change in permeability is much larger for those particles that lie on the interface.

For a Hele-Shaw cell, the permeability is proportional to the square of the plate spacing $b$. Let $b_{0}$ be the average value of $b$ and let $b_{1}$ be the spatiallyvarying uniformly-small deviation of $b$ from its average. That is, we assume $\left|b_{1}(x, y)\right| \ll b_{0} \ll R$ where $R$ is a representative value of the radius of curvature of the interface in the plane of the cell. The inequality on the right is a general requirement of the Hele-Shaw approximation. Note that $R$ scales as the critical wavelength for instability of the front which varies, in turn, as $L \tau^{1 / 2}$. In addition, the requirement of small slope of the cell wall implies that gradients associated with permeability variation are $O(1 / R)$. For variable permeability, the field equation is

$$
\nabla \cdot\left(b^{2} \nabla p\right)=0 .
$$

Expanding equation (23) for small permeability variation and letting $p=p_{0}+p_{1}$, we find that $p_{0}$ satisfies Laplace's equation while $p_{1}$ satisfies the inhomogeneous equation $\nabla^{2} p_{1}=-\left(2 / b_{0}\right) \nabla b_{1} \cdot \nabla p_{0}$. This Poisson equation sets the scale of the perturbation pressure gradient $\nabla p_{1}$. Because the mean value of $b_{1}$ is zero, the perturbation pressure gradient is

$$
\left|\nabla p_{1}\right| /\left|\nabla p_{0}\right| \sim O\left(b_{1} / b_{0}\right)
$$

at most.

The situation is quite different for points on the boundary. When the plate spacing is allowed to vary, the boundary condition (6) must be replaced by

$$
p=\sigma[(1 / b)+(1 / R)], \quad(x, y) \in \partial D .
$$

The first term in parentheses is much larger than the second, but when the plate spacing is strictly constant, its gradient is zero and it may be ignored. With $b$ 
variable however, we have, upon expanding the right side of (22a) for small $b_{1}$, that $\left|\nabla p_{0}\right| / \sigma \sim O\left(R^{-2}\right)$ and $\left|\nabla p_{1}\right| / \sigma \sim O\left(b_{1} b_{0}^{-2} R^{-1}\right)$. Consequently

$$
\frac{\left|\nabla p_{1}\right|}{\left|\nabla p_{0}\right|} \sim O\left(\frac{R}{b_{0}}\right)\left(\frac{b_{1}}{b_{0}}\right)
$$

and we have established that, because of the capillary pressure jump mechanism in a Hele-Shaw cell, small variations in permeability influence the solution most strongly through the change in the boundary condition. In terms of their relative influence on the induced velocity field, we see, by comparing equations (24) and $(25 \mathrm{~b})$, that boundary permeability variations are more important by a factor of $R / b_{0}$. The only exception to this conclusion is the uninteresting case when the interface is uniformly stable in space for the entire flood history.

For the value $\tau=0.001$ and $R_{0}=0.1$, a number of cases, corresponding to various values of noise level, were run. Provided $\delta$ was less than $0\left(10^{-5}\right)$, flood histories similar to Figure 3 were obtained. For larger values of $\delta$, pronounced asymmetry resulted; a variety of different types of profiles, exhibiting quite different sweep efficiencies, could be produced for the same value of $\delta$. Two such cases, both for $\delta=.0001$ are shown in Figure 4. In (4a) the flood front breaks up quite evenly about the centreline yielding, thereby, a large recovery. In (4b), on the other hand, a recess develops to one side and the recovery is reduced.

In all our numerical experiments, the fingering pattern is established quite early in the flood. Similarly, both Habermann [7] and Lee \& Claridge [11], in their experiments, also observe early breakup. This provides justification for our simple stability analysis that only considers competition between noise and stable growth on the initial profile. Our particular choice for $A$, a random number

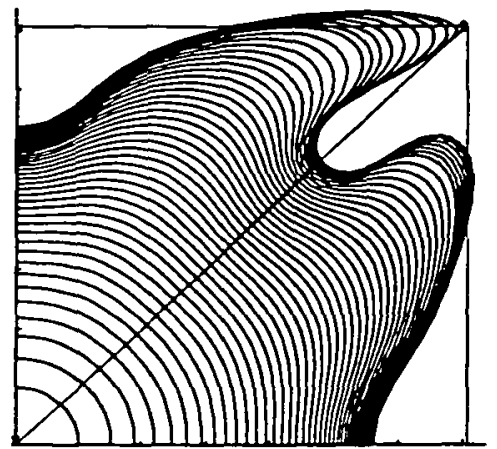

(a)

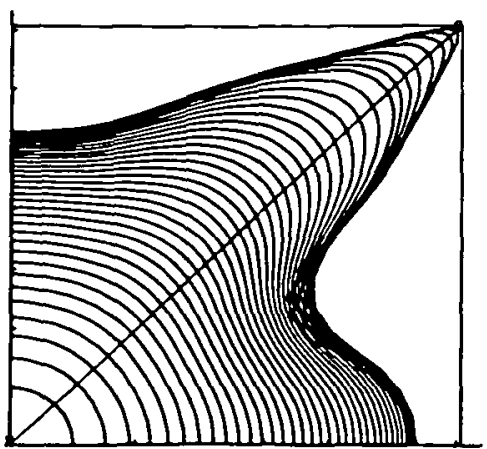

(b)

Figure 4. Noise-induced pattern selection for $\tau=0.01, \Delta t=0.01$; (4a) the sweep efficiency is $84.2 \%$; the efficiency in (4b) is $68.8 \%$. For both cases the noise level is equivalent to a $0.5 \%$ permeability variation. 
between -1 and 1 , corresponds to white noise where the expected magnitudes of all resolvable Fourier coefficients are the same and are equal to $(12 N)^{-1 / 2}$. Here $N$ is the number of points that are uniformly distributed on the initial quarter circle. The critical value of $\delta$ for each noise mode, $\delta_{j}^{*}$ say, is given approximately by

$$
\delta_{j}^{*}=(3 N)^{1 / 2} \pi \hat{R}_{0} \hat{\varepsilon}_{j}^{*}=(3 N)^{1 / 2} \pi \hat{R}_{0}^{6}\left\{(j-1)\left[1-\pi j(j+1) \tau /\left(2 \hat{R}_{0}\right)\right]\right\}^{-1}
$$

where equations (20) and (21) have been used. For $\tau=0.001, r_{0}=0.1$ and $B=0.75$, equation (26) predicts values of $\delta^{*}$ equal to $0.000025,0.000011$, and 0.000013 for modes 2,4 , and 6 , respectively. Mode 8 and higher modes are all stable. Returning to Figure 4, we see that (4a) appears to have a mode 4 disturbance, while (4b) grows from a disturbance mode equal to 6 . The predicted magnitudes of $\delta^{*}$ also appear to be consistent with the numerical results as will be demonstrated.

We have also extended our numerical results to lower values of $\tau$. Starting from a small initial circle, we find that a stable flood pattern cannot be produced. This is consistent with our findings for the linear channel [5], [6] at small $\tau$. We attribute this to an ambient noise level in the algorithm associated with the discrete approximation of continuous functions. Both the spline redistribution scheme and the numerical treatment of the improper integrals in equations (9) are particularly implicated. In order to produce a determinate result however, we introduce a particular perturbation that, while small, is still much larger than the algorithmic noise. Figure 5 shows the early and late portions of the flood history for $\tau=5 \times 10^{-5}$ using the initial profile

$$
R_{0}(\theta)= \begin{cases}0.1+A[1+\cos 12(\theta-\pi / 3)], & |\theta-\pi / 3| \leq \pi / 12 \\ 0.1, & |\theta-\pi / 3|>\pi / 12\end{cases}
$$

A small bump, using $A=0.001$, imperceptible in Figure 5a, is located near the asterisk on the $R_{0}$ profile. The disturbance grows quickly and, because it possesses components of all the Fourier modes, provides, in effect, an initial disturbance for each of them. By the time the third front in the figure is reached, it is clear that three and a half maxima are produced in the quadrant. This corresponds to mode 14 on the full circle. Mode 14 is, in fact, the one with the largest growth coefficient as given by equation (14). Profiles up to breakthrough are shown in Figure (5b). Notice that each finger, except the central one, ultimately stops growing, with the ones furthest from the producer stopping first. For the essentially stagnant fingers further from the diagonal, there is a tendency for them to slowly reduce the length of their boundaries through the action of surface tension. By comparing Figures 4 and 5, we see that the finestructure increases as $\tau$ is reduced, in this case, by a factor of 20 . If we define the fine-structure as the number of inflection points on the breakthrough profile, 

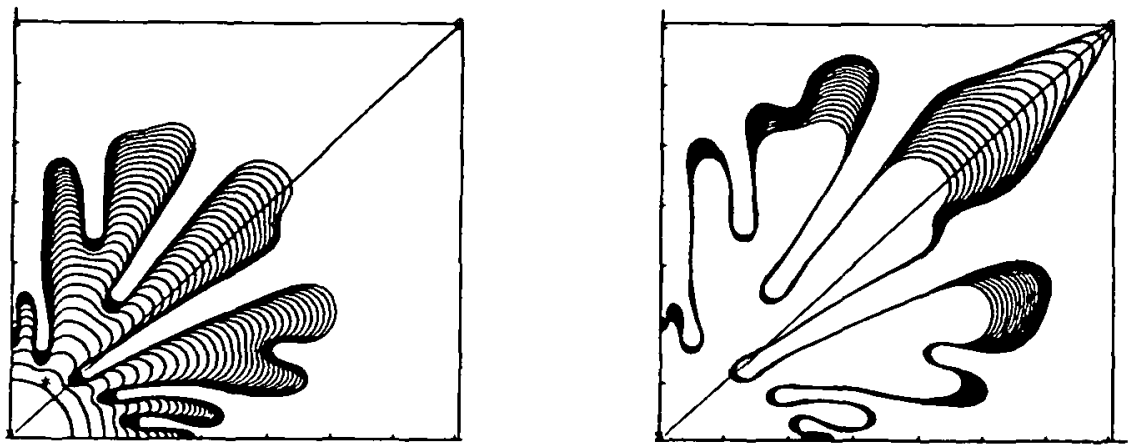

Figure 5. Multiple fingering caused by placing a small disturbance on initial circle near the asterisk, $\tau=5 \times 10^{-5}$. Early and late frontal profiles; $\Delta t=0.005$.

for example, on each figure, it is apparent that this increase scales essentially as $\tau^{-1 / 2}$, as would have been predicted by the linear stability analysis given above.

In Figure 6 we show front profiles from the miscible experiments of Lee \& Claridge [11] and Habermann [7]. In both of these, the viscosity ratio is such that the floods are quite unstable. While these experiments also differed from our simulation in that a developed five-spot geometry was employed, there are a number of similarities with our results in Figure 5. In each of these studies the almost immediate breakup can be attributed to the viscous fingering instability. As in Figure 5, the competition mechanism leads to a single finger moving towards a diagonal line between injector and producer with more remote branches moving only slowly. In both cases the sweep efficiency is lower than our result, 48.6 per cent for the case shown in Figure 5, but this is also consistent with the observation that the experiments show breakup into more fingers initially. In the experiments the stabilizing mechanism is thought to be diffusive mixing and there is no reason why this effect should be a detailed analog for surface tension in a Hele-Shaw experiment or simulation. We hope soon to have the capability of running at much smaller $\tau$ values; it should then be possible to measure and compare quantitative features of the two processes. It is clear, incidentally, in Habermann's experiments, that the front is not breaking up on the pore scale. For the mesh size of the sand used, the sand particles are smaller than the scale of the narrowest fingers by perhaps a factor of 100 . The value of $\tau$ used for Figure $5,5 \times 10^{-5}$, is our current practical lower limit on this quantity using the present algorithm. This calculation used a maximum of 313 points to describe the profile and required about five hours of computation time on the IBM 3033 computer.

In order to assess the effect of noise in a systematic manner, a number of runs were made, for several different values of $\delta$ using $\tau=10^{-3}$. The sweep efficiency 

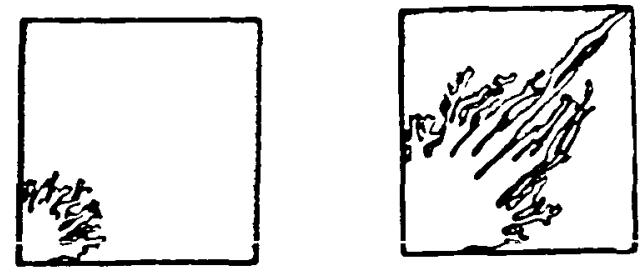

(a)

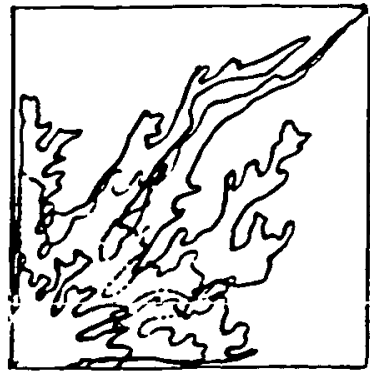

(b)

Figure 6. Laboratory floods in a developed 5-spot at high mobility ratio $M$.

(a) Miscible Hele-Shaw cell, $M=10, \eta=0.40$ (Lee \& Claridge, 1968).

(b) Miscible sand-pack, $M=17.3, \eta=0.3$ (Habermann, 1960).

results, for ten runs at each $\delta$ value, are shown in Table II. Also given are the corresponding permeability variations using equation (22).

TABLE II. Dependence of Sweep Efficiency on Noise Level. Statistics of 10 runs for each value of $\delta, \tau=0.001$

\begin{tabular}{|c|c|c|c|c|c|}
\hline \multirow[t]{2}{*}{$\delta$} & \multirow{2}{*}{$\begin{array}{c}\langle\delta k / k\rangle \\
(\%)\end{array}$} & \multicolumn{3}{|c|}{ Sweep Efficiency (\%) } & \multirow{2}{*}{$\begin{array}{c}\text { Standard } \\
\text { deviation } \\
(\%)\end{array}$} \\
\hline & & $\operatorname{Max}$ & $\mathrm{Mi}$ & Mean & \\
\hline $10^{-6}$ & 0.005 & 73.7 & 73.5 & 73.6 & 0.08 \\
\hline $10^{-5}$ & 0.05 & 74.7 & 72.8 & 73.7 & 0.57 \\
\hline $10^{-4}$ & 0.5 & 84.2 & 68.4 & 72.7 & 4.7 \\
\hline $2 \times 10^{-4}$ & 1.0 & $\mathbf{7 7 . 6}$ & 65.4 & 69.3 & 4.0 \\
\hline $4 \times 10^{-4}$ & 2.0 & 83.1 & 62.5 & 71.0 & 7.1 \\
\hline $8 \times 10^{-4}$ & 4.0 & 70.5 & 59.8 & 64.8 & 3.8 \\
\hline
\end{tabular}

We see, in this table, that for very small noise, the sweep efficiency results are essentially constant at the deterministic value 73.6 per cent. When the noise level is increased to $10^{-5}$, the numerical results become scattered about their mean value to a significant degree. Recall that $\delta=10^{-5}$ is the predicted critical noise level using the simple competition model. As the noise increases further, the scatter also increases until $\delta=O\left(10^{-4}\right)$. At this point further noise increase cannot be said to have either a quantitative or qualitative effect. For this value of $\delta$, there appears to be only a small average reduction in sweep efficiency as $\delta$ increases past its threshold value.

The most striking effect in these noisy calculations is the very small value of threshold noise. For a Hele-Shaw cell, spatial variations in plate spacing of less than one per cent are quite sufficient to excite flow patterns of the types shown in Figure 4. To the extent that we interpret noise as due only to permeability variation, our model presupposes that flow paths of small resistance are uncorrelated over length scales equal to travel distances between point-redistribution 
time intervals. That is, we assume that important continuous high-permeability paths between injector and producer are not present. Yet we see that small permeability heterogeneity of this type can result in gross alteration in the flooding history. While this effect produces a small decrease in sweep efficiency, on average, for this value of $\tau$, more modes are excited at smaller $\tau$ with significant reduction in sweep efficiency, as in Figure 5.

In Figure 7 we show the dependence of areal sweep efficiency on $\tau$. Data are given for values of $\tau$ between $5 \times 10^{-4}$ and 0.5 . Shown for reference purposes is the limiting efficiency for two-phase flow when $\tau$ goes to infinity. In that case the breakthrough profile is simply a circle of unit radius. Recall that, for single-phase flow, $\eta$ is 105 per cent. The solid curve includes results from 12 runs that exhibit flooding histories without finger splitting. Stable fingers could not be produced, starting from a circle of radius 0.1 for $\tau$ less than $3 \times 10^{-4}$. We see that $\eta$ decreases rather slowly as $\tau$ is reduced. Also shown, as a dashed line in the figure, are results obtained when the initial profile contains the small bump as given by equation (27). The effect of this disturbance on $\eta$ is quite pronounced for small values of $\tau$. In all cases tested, with this bump, efficiencies are lower than for the stable patterns; because the imposed disturbance is very small, perhaps comparable to imperfections in a laboratory Hele-Shaw cell, this dashed curve is expected to be more representative of experimental results at low $\tau$ values. For relatively large $\tau$, on the other hand, the modes excited by this initial disturbance quickly decay. Thus the bump is seen to have negligible effect for $\tau>0.005$.

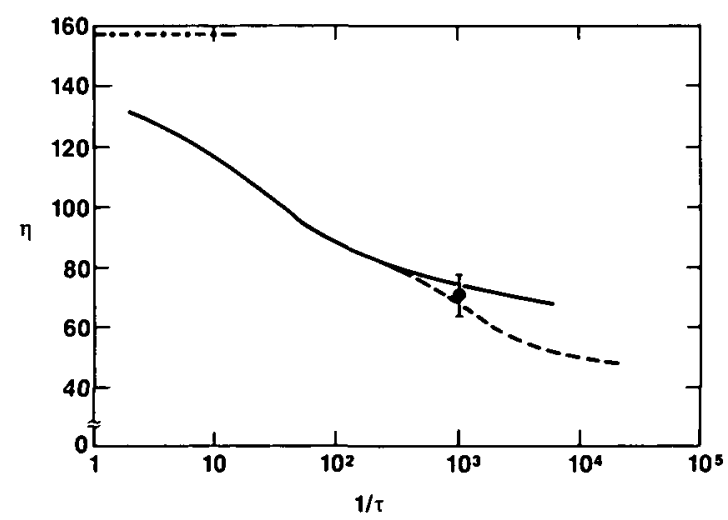

Figure 7. Sweep efficiency versus modified capillary number $\tau^{-1}$ stable solutions; $\ldots$ - results with perturbed initial profile given by equation (24); for $\tau=10^{-3}$, mean value and standard deviation, for 10 runs at noise level $\delta=4 \times 10^{-4}$, are shown. 
Results of runs made with noise for $\tau=10^{-3}$ are also shown in Figure 7 . The two per cent permeability variation result, from Table II, is presented as an average value with standard deviation error bars using the 10-run sample. Note that the magnitude of the error bars is consistent with the difference between the stable and perturbed results for this value of $\tau$.

\section{Fingering patterns at finite viscosity ratio}

In this section we present a technique for the numerical calculation of twophase displacement, using the Hele-Shaw equations, when the ratio of driver to driven fluid viscosities, equivalent to the reciprocal of the mobility ratio, is finite. As before, we use a boundary integral scheme with continuously-adjustable point spacing. For the present more general problem, we have modified the technique so as to use a singular vorticity distribution on the interface rather than a source distribution. Inspection of the interface conditions for the more general problem reveals that, for an interface represented by $N$ line segments, a source method leads to an imbedded linear problem of dimension $2 N$, while the vorticity method yields a system of dimension $N$. The new method has been validated by comparing results with the source-distribution algorithm for $m=0$.

As before, two rather distinct types of solutions will be presented. The first, which we refer to as stable solutions, is characterised by a smooth interface where the only asymmetry results from the suction of the remote sinks. Such solutions display relatively large values of $\eta$, the breakthrough sweep efficiency. When these solutions are sufficiently perturbed, however, the displacement front breaks up into a multiply-fingering pattern; this second type of solution typically corresponds to smaller values of $\eta$. Because the required noise level is very low, these unstable solutions will be the ones observed experimentally for systems with low interfacial tension or, equivalently, at high flooding rates.

A vortex distribution $\omega(s)$ on $\partial D$ is assumed whose strength is determined as part of the solution. With this singularity distribution, condition (4b) is satisfied identically, while the tangential velocity discontinuity on $\partial D$ is simply equal to the local value of $\omega$. The vorticity distribution satisfying (2), (3), and (4) is the solution of the singular second-kind Fredholm equation

$$
\begin{gathered}
\frac{(1+m)}{2} \omega\left(z_{0}\right)+(1-m) \operatorname{Re}\left[\frac{i \exp \left(i \alpha_{0}\right)}{2 \pi} \int \frac{\omega(s) d s}{z-z_{0}}\right] \\
=\frac{\tau d \kappa}{d s_{0}}-(1-m) \operatorname{Re}\left[\frac{d f}{d z_{0}} \exp \left(i \alpha_{0}\right)\right]
\end{gathered}
$$

where the integral is to be interpreted as a Cauchy principal value and Re denotes the real part of a complex function. The dimensionless surface tension parameter 
is now referenced to the viscosity of the displaced phase, that is

$$
\tau=\left(\sigma b^{2}\right) /\left(12 \mu_{1} Q L\right) .
$$

Equation (28) is obtained by differentiating (4a) in the tangential direction and replacing the velocities in favour of the vorticity distribution. $\alpha_{0}$ is the angle subtended by a tangent vector to the interface and the positive $x_{0}$ axis. The function $f\left(z_{0}\right)$ is the complex potential for the single-phase source-sink flow given by (12). Once the vorticity distribution has been determined, the normal velocity of points on the boundary is given by

$$
\frac{\partial \phi}{\partial n}=-\operatorname{Im}\left[\frac{d f}{d z_{0}} \exp \left(i \alpha_{0}\right)+\frac{i \exp \left(i \alpha_{0}\right)}{2 \pi} \int \frac{\omega(s) d s}{z_{0}-z}\right] .
$$

The vortex method given here is applicable to general plane flows with discrete sources and sinks. As before, we discuss only five-spot flows with reflectional symmetry in each coordinate axis. Numerically, the algorithm includes the following features:

(a) The time integration in condition (4c) is done implicitly using the LSODE [8] package.

(b) The discretization of equations (28) and (30) employs a method that is equivalent to separating the singular and nonsingular parts of the Cauchy principal value integral. The singular part is integrated analytically. The remainder is treated by assuming the vorticity to be piecewise linear. Certain other details are similar to the procedures discussed by Moore [14]; however the point spacing along the interface is determined by local stability considerations and simplifications possible with equally-spaced points are inapplicable here.

(c) Points are periodically redistributed along a cubic spline representation of the interface so as to provide resolution of all locally unstable perturbations, using the linear stability result given by Saffman \& Taylor [19]. That is, for the unstable cases, corresponding to $m<1$, the point spacing is no greater than

$$
\Delta s=B\left[\tau /\left(v_{n}(1-m)\right)\right]^{1 / 2}
$$

where $B$ is a constant and $v_{n}$ is the normal component of velocity locally. Closer point spacing is used when necessary in regions of large interface curvature.

(d) The linear algebraic system corresponding to equation (28) is solved by Gauss elimination. While implicit time integration and Gauss elimination minimize numerical errors associated with these processes, computational costs are relatively high. Since the linear algebraic system equivalent to equation (28) is diagonally dominant, potential savings can be realized by use of an iterative solver such as a Neumann series expansion [3].

The algorithm has been used to generate flood histories for a variety of values of surface tension and viscosity ratio. Two such cases are shown in Figures (8a) and $(8 \mathrm{~b})$. Both of these are for $\tau=2 \times 10^{-4}$ with an initial profile taken to be a 
circle of radius 0.1 . Figure (8a) presents results for the favorable viscosity ratio $m=5$. The point redistribution time interval is taken as 0.01 ; so as to view successive fronts more distinctly, we show profiles spaced by 0.05 times units only. Since the front is locally stable, the point spacing is taken to be constant using $\Delta s=0.025$ except in regions of high curvature, near breakthrough, when more points are added. Observe that the flood proceeds essentially as an expanding circle until it is close to the sink, when a cusp begins to form. In the limiting cases, corresponding to arbitrarily large viscosity ratio and/or surface tension, the flooded area should be a circle with radius equal to the well spacing. This limiting value, $\pi / 2$, may be compared with the calculated sweep efficiency for the present case, which is 131 per cent.

At the other end of the stability range, we show, in Figure (8b), results for the unfavorable viscosity ratio $m=0.1$. Time intervals between point redistribution

(a)

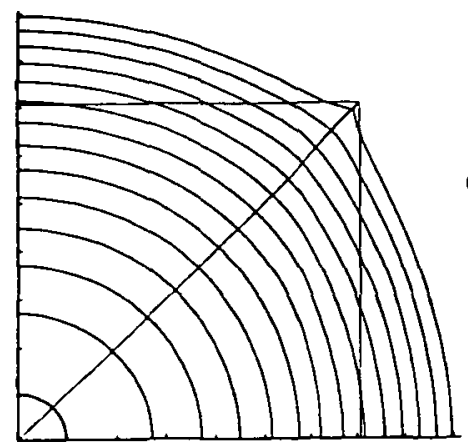

(b)

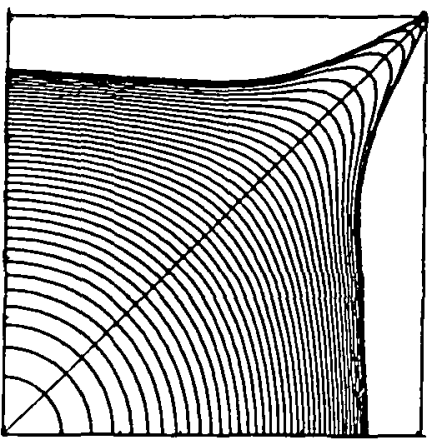

(c)

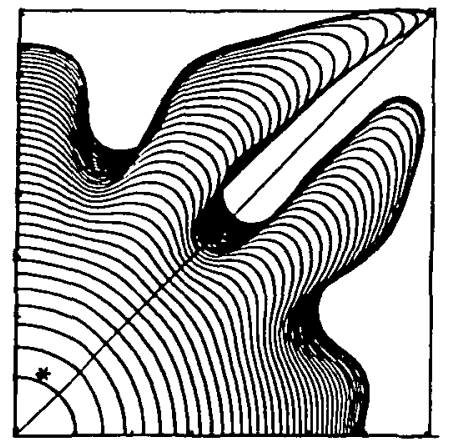

Figure 8. Three floods at $\tau=2 \times 10^{-4}$.

(a) $m=5.0, \eta=131 \%$.

(b) $m=0.1 ; \eta=75.6 \%$.

(c) The same conditions as for figure (8b), except a small bump, given by eqn. (10) with $A=10^{-5}$, is imposed on the initial profile; $\eta=71.1 \%$. 
were taken as 0.01 except for the last five profiles shown. When the displacement front was close to the sink, points were redistributed more frequently so as to maintain high resolution when the surface is dilating rapidly. The spacing constant $B$ in equation (9) was taken as 0.75 , corresponding to about nine points per neutrally-stable wavelength using local conditions. Symmetry about the line connecting source and sink was not imposed a priori in this calculation. Each of the output profiles shown here was quite symmetric however; the horizontal and vertical intercepts, for example, differed in all cases by less than one part in $10^{-3}$.

The case shown in Figure (8c) uses parameter values indentical to those in (8b), except that a very small bump is placed on the initial profile near the asterisk. The resulting profile has continuous slope; its equation is given by (24) with $A=10^{-5}$. Because of the small value of $\tau$, the time history of the flood is exceedingly sensitive to the initial conditions. A gross alteration of the flow develops that is characterized, ultimately, by two competing fingers heading for the sink.

In previous sections, we considered only floods where the driver viscosity was zero. For the multiply-fingered patterns in Figures 4 and 5, troughs, once left behind, remain essentially stagnant. Here, even though the viscosity ratio is only 0.1 , the troughs continue to move forward at a speed which is an appreciable fraction of the speed of the neighboring crests.

In Figure 9 we show the dependence of $\eta$ on the disturbance amplitude $A$, from equation (24), for three combinations of surface tension and viscosity ratio. The

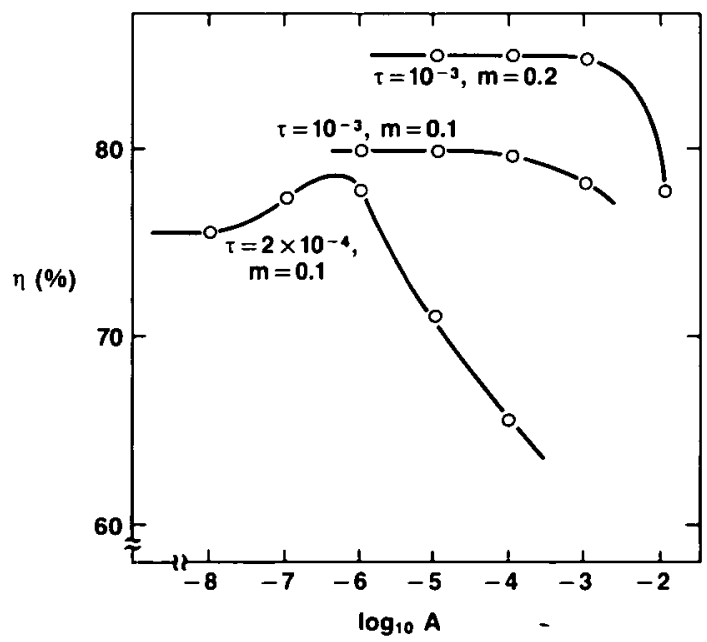

Frgure 9. Variation in sweep efficiency $\eta$ with disturbance amplitude $A$ for three combinations of $\tau$ and $m$. 
purpose of this comparison is the prediction of the critical disturbance amplitudes required to alter stable sweeps, such as that of Figure (8a). For $\tau=2 \times 10^{-4}$ and $m=0.1, \eta$ remains constant at the undisturbed value, 75.6 per cent, until $A$ exceeds $10^{-8}$. Interestingly, $\eta$ first increases with increasing $A$ before ultimately decreasing. (This somewhat surprising result is due to the excitation of modes that tend to flatten the flood front for this particular range of values of $A$ and $\tau$.) Holding $m$ constant, we increase $\tau$ by a factor of five to $10^{-3}$. The critical value of $A$ increases by almost four orders of magnitude to about $10^{-4}$. Holding $\tau$ fixed, $m$ is now increased by a factor of two. The value of $A$ required to produce a small but noticeable change in $\eta$ increases by perhaps an additional order of magnitude.

For finite viscosity ratio, we thus demonstrate numerically the existence of linear stability, but nonlinear instability of a five-spot flood. In the absence of any disturbance, the flood is symmetric and characterized, usually, by relatively high values of $\eta$. A small disturbance may destabilize the flood: the critical magnitude of the required disturbance decreases dramatically with reduction in surface tension or increase in flooding speed. Somewhat less dramatic, but still important, is the effect of changing the viscosity ratio while remaining in the unstable regime. In our previous work, we investigated this question, for zero viscosity ratio, for both the rectilinear channel [5], [6] and the five-spot pattern (Section 4). We see that the nonlinear character of the instability established there, and driven by random noise, is also pertinent to finite viscosity ratio cases.

The exceedingly low values of the critical disturbance amplitude suggest that experiments run in the small $\tau$ regime will undoubtedly exhibit tip splitting. In addition to the miscible flood of Lee \& Claridge [11] shown in Figure 6, recent experiments by Park \& Homsy [16] using immiscible fluids in a rectilinear HeleShaw cell have also shown tip splitting. We believe that these experimental results are manifestations of the same nonlinear effect and that this effect is modelled, at least qualitatively, by the Hele-Shaw system used here. In his monograph on oil-reservoir water flooding, Craig [4] compiles sweep efficiency data, obtained in ten laboratory investigations that use a variety of techniques. These include miscible and immiscible floods in sand and bead packs, resistance networks, and electrolytic and potentiometric models; see especially his Figure 5.2. Craig notes that there is "satisfactory agreement among most investigators when the mobility ratio is $\mathbf{1 . 0}$ or less. However, at mobility ratios above unity a wide divergence between reported values occurs." He offers no explanation for this divergence.

In Figure 10, we present calculated values of $\eta$ versus mobility ratio for 6 values of $\tau$. Results for $\tau=0$ are included, but only for $M<1$. Local stability considerations suggest, and our numerical experiments confirm, that the zero-surface- tension problem is completely ill-posed when the viscosity ratio 


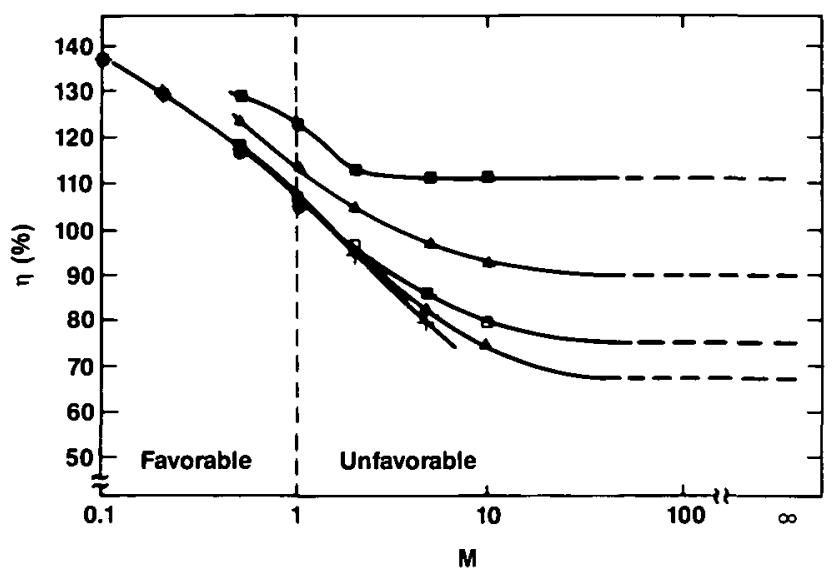

Figure 10. Sweep efficiency versus mobility ratio $M$, for an isolated five-spot, using present method. Results are presented for 6 different values of inverse capillary number $\tau$.

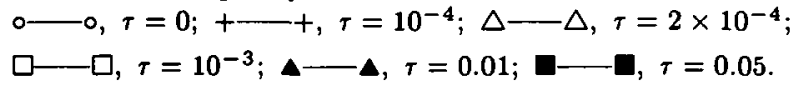

is unfavourable. We observe, in our results, relatively minor dependence of $\eta$ on $\tau$ in the stable range. Much more pronounced divergence is seen as $M$ is increased. For the mathematical model, the control parameter $\tau$, an inverse capillary number, is a measure of the speed of the displacement process. We suggest that such a speed dependence explains the divergence of the data presented by Craig. Because Craig's data is compiled for a "developed" five-spot, that is for a doubly-periodic pattern, the actual numerical range of $\eta$ is different. If the swept areas, in both cases, are referenced to the appropriate single-phase results $(m=1, \tau=0)$, which is 72 per cent for the developed and 105 per cent for the isolated five-spots respectively, a quite reasonable overlap of the data range can be obtained.

\section{Concluding remarks}

In this paper we have presented results for the flooding history of an isolated 5 -spot pattern as calculated by integration of the Hele-Shaw equations. We have demonstrated the linear stability of smooth profile solutions over several orders of magnitude in the single independent parameter $\tau$, an inverse capillary number. When sufficient noise is introduced into the system, the stable solutions break down and are replaced by patterns that exhibit many competing fingers. 
The amount of noise required to destabilize the symmetrical patterns decreases rapidly as $\tau$ is reduced. In this regard the effect is entirely equivalent to our findings [5], [6] for the rectilinear channel. In the five-spot geometry, the breakup mechanism leads to a reduction in sweep efficiency, the magnitude of the reduction becoming greater as $\tau$ is made smaller. This effect was demonstrated by inserting a small bump on the initial profile and determining the resulting function $\eta(\tau)$ as shown in Figure 7.

Rather than use a specific disturbance, we have also made runs where random white noise was introduced into the calculation to simulate permeability variation. Once the threshold noise level is exceeded, there may no longer be a strong dependence of $\eta$ on its actual magnitude. Rather we suspect that low-level noise here acts as a trigger to cause the flood to enter the competing-finger regime. In this regime, the value of $\eta$ is determined largely by the minimum finger width, the latter scaling as $\tau^{1 / 2}$. More statistical evidence, of the type presented in Table II, is required to validate this premise, however.

Because the noise in our model is not spatially correlated, this type of heterogeneity is qualitatively different from heterogeneity that leads to connected high-permeability paths, the latter being a type of "percolation" concept. In order to fit network models to observed laboratory measurements of $\eta$, often an unrealistically high degree of heterogeneity must be assigned to the permeable medium. This has been pointed out by Claridge [2] in his criticism of earlier theoretical work [20], [21]. His suggestion that the missing ingredient is the viscous fingering instability is borne out by our numerical work.

At present our specific predictions of the dependence on $\eta$ on $\tau$ cannot be compared with experiment since this particular parameter range has yet to explored in the laboratory. Such cases should not be difficult to investigate experimentally. For a flood at $\tau=.001$ in a cell measuring $30 \mathrm{~cm}$ on a side with a plate space of $1 \mathrm{~mm}$, values of $\sigma=50$ dynes $/ \mathrm{cm}, \mu=1$ poise, and a volumetric flow rate of $0.1 \mathrm{cc} / \mathrm{sec}$, the time to breakthrough will be about 15 minutes.

The Hele-Shaw model with surface tension may be capable of simulating existing miscible experiments [11], [12] with a suitable small value of $\tau$. The experiments shown in Figure 6 possess a fine structure that is perhaps a factor of three smaller than our computation shown in Figure 5. In order to effect such a comparison, it would be necessary to solve for fronts whose description may require a thousand points or more. This is presently not possible using direct Gauss elimination to solve the linear integral equations in (9a) or (28). On the other hand, an iterative solution strategy can be formulated and should provide an increased low- $\tau$ capability.

Lower values of sweep efficiency than those calculated here would have been obtained if four-fold symmetry in the flooding pattern had not been imposed $a$ 
priori. One reason for this is that the symmetry assumption rules out half of the potentially unstable modes. Four identical streams, each heading towards a production well, is an unstable situation. As soon as one stream gets ahead of the others, its growth will be enhanced at the expense of its competitors. This would be particularly true if the flood were run at constant pressure drop between injector and producers rather than at constant volumetric flow rate as we have done here. It may be possible to mitigate this effect by restricting or stopping the production at the well corresponding to the furthest advance to the front. Such an optimization is possible using the present methods and the results are potentially useful.

It is clear that detailed resolution of fine structure at scales comparable to oil reservoir floods will never be possible. If we assume $100 \mathrm{~m}$ well-spacing and single-phase permeability of 1 Darcy $\left(\approx 10^{-8} \mathrm{~cm}^{2}\right)$ with typical values of oilwater interfacial tension, the corresponding value of $\tau$ is of order $10^{-11}$. However the large range of length scales in porous media problems is certainly suggestive of the existence of scaling laws and self-similar behavior. With relatively minor computational improvements, it may be possible to investigate this question and determine possible power-law dependence of sweep efficiency on the parameter $\tau$.

The present Hele-Shaw model assumes that the pressure difference at the front separating two immiscible phases is proportional to the interface curvature. When applied to porous media, it is necessary to relate this (capillary) pressure difference to quantities that are macroscopic when measured in units of a typical pore dimension. While experimental evidence has been offered that selection of an effective surface tension that is a constant multiple of the real surface tension produces an acceptable fit to core flood data [1], [18], it is generally believed that this simple viewpoint is inadequate. Recently Jerauld, Davis and Scriven [9] have developed a model for analyzing the linear instability of a slightly perturbed plane interface that incorporates information about pore-level processes. Their model also exhibits a short-wavelength cutoff as we believe any self-consistent theory must do. It would be possible to insert a different jump condition at the front into our nonlinear algorithm and to determine the effect of such a modification upon the fingering behavior.

A problem that is closely related to two-phase Hele-Shaw flow and may be similarly idealized is dendritic growth in solidification [23]. In fact both problems are used as examples of pattern formation in nonlinear systems [10], [13]. Under a different set of assumptions, pattern formation problems have been treated by the technique known as diffusion limited aggregation [25]. A comparison of those predictions with the results of our boundary-integral front-tracking procedure would seem worthwhile. 


\section{References}

[1] R. L. Chouke, P. van Meurs and C. van der Poel, Trans. AIME 216 (1959), 188-194.

[2] E.L. Claridge, Soc. Pet. Eng. J. (1972), 352-361.

[3] R. Courant and D. Hilbert, Methods of Mathematical Physics, Vol. 1 (Interscience, New York, 1953).

[4] F. F. Craig, The reservoir engineering aspects of waterflooding (Soc. Pet. Eng., New York, 1971).

[5] A. J. DeGregoria and L. Schwartz, Phys. Fluids 28 (1985), 2313-2314.

[6] A. J. DeGregoria and L. W. Schwartz, J. Flutd Mech. 164 (1986), 383-400.

[7] B. Habermann, Trans. AIME 219 (1960), 264-272.

[8] A. C. Hindmarsh, "Livermore Solver for Ordinary Differential Equations" (LSODE), Lawrence Livermore Laboratory, Livermore, Ca., USA. Sept. 23, 1980 version.

[9] J. R. Jerauld, H. T. Davis and L. E. Scriven, SPE/DOE Preprint No. 12691, U.S. Dept. Energy, (1984).

[10] D. A. Kessler, J. Koplik and H. Levine, Phys. Rev. A 30 (1984), 3161.

[11] K. S. Lee and E. L. Claridge, Soc. Pet. Eng. J. (1968), 52-62.

[12] J. Mahaffey, W. Rutherford and C. Matthew, Soc. Pet. Eng. J. (1966), 73-80.

[13] B. B. Mandelbrot, The Fractal Geometry of Nature (Freeman, San Francisco, 1982).

[14] D. Moore, "Numerical and analytical aspects of Helmholtz instability", Proc. IUTAM (1985), 263-274.

[15] M. Muskat, The flow of homogeneous fluids through porous media (McGraw-Hill, New York, 1937).

[16] C. W. Park and G. M. Homsy, Phys. Fluids 28 (1985), 1583.

[17] L. Paterson, J. Fluid Mech. 113 (1981), 513-529.

[18] E. Peters and D. Flock, Soc. Pet. Eng. J. (1981), 249-258.

[19] P. G. Saffman and G. I. Taylor, Proc. Roy. Soc. London Ser. A245 (1958), 312-329.

[20] R. Simon and F. Kelsey, Soc. Pet. Eng. J. (1971), 99-112.

[21] R. Simon and F. Kelsey, Soc. Pet. Eng. J. (1972), 345-351.

[22] G. Tryggvason and H. Aref, J. Fluid Mech. 136, (1983), 1-30.

[23] L. H. Ungar and R. A. Brown, Phys. Rev. B 29(3) (1984), 1367-1380.

[24] S. D. R. Wilson. J. Colloid Interf. Scr. 51 (1975), 532-534.

[25] T. A. Witten and L. M. Sander, Phys. Rev. B 27 (1983), 5686. 